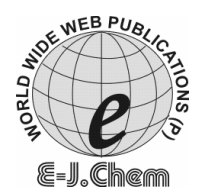

http://www.e-journals.net
ISSN: 0973-4945; CODEN ECJHAO

E-Journal of Chemistry

2009, 6(3), 830-834

\title{
Thermal Degradation Study of Salicylic Acid, Diaminonaphthalene and Formaldehyde Terpolymer
}

\author{
DHANRAJ T.MASRAM \\ Department of Chemistry, \\ Rashtrasant Tukdoji Maharaj Nagpur University, Nagpur-440033. \\ dhanraj_masram27@rediffmail.com
}

Received 23 October 2008; Accepted 12 December 2008

\begin{abstract}
The terpolymer resin salicylic acid-diaminonaphthalene-formaldehyde (SDNF) has been synthesized by the condensation of salicylic acid and diaminonaphthalene with formaldehyde in the presence of catalyst hydrochloric acid in 1:1:2 molar proportions of reactants. Detailed thermal degradation studies of the SDNF terpolymer resin has been carried out to ascertain its thermal stability. Thermal degradation curve has been discussed in order to determine their mode of decomposition, order of reaction, apparent activation energy, frequency factor, free energy change, entropy change, and apparent energy change. Freeman - Carroll and Sharp- Wentworth methods have been applied for the calculation of kinetic parameters while the data from the Freeman - Carroll methods have been used to determine various thermodynamic parameters.
\end{abstract}

Keywords: Degradation, Order of reaction, Entropy change, Apparent energy change.

\section{Introduction}

Phenolic resins have a large number of practical applications in electronic controls, insulating materials, protective adhesives, aerospace industries etc. because of their high thermal stability, heat and chemical resistance and electrical insulation properties ${ }^{1}$. Various researchers have been studied the applications of terpolymer resins of substituted phenols and formaldehyde $e^{2,3}$. Terpolymers of salicylic acid, thiourea with trioxane and $p$-hydroxybenzoic acid, thiourea with trioxane have been reported in the literature ${ }^{3-7}$. Manavalan and $\mathrm{Patel}^{8}$ synthesized resins of salicylic acid, urea and formaldehyde. In our laboratory, extensive research work has been carried out on synthesis, characterization and thermal degradation of terpolymers of salicylic acid, $p$-hydroxybenzoic acid, diamide, urea, thiourea with formaldehyde $\mathrm{e}^{1,5-7,12,13}$.

The present communication deals with synthetic and thermal degradation properties of a newly synthesized terpolymer resin derived from salicylic acid, diaminonaphthalene and 
formaldehyde. The Freeman - Carroll and Sharp- Wentworth methods have been applied for the calculation of kinetic parameters ${ }^{9-11}$. Methods for the estimation of kinetic parameters from thermogravimetric studies are generally based on the assumption that the Arrhenius equation is valid with thermal and diffusion barriers are negligible.

\section{Experimental}

\section{Synthesis of salicylic acid - diaminonaphthalene -formaldehyde terpolymer resin}

A mixture of salicylic acid, diaminonaphthalene, and formaldehyde in the molar ratio 1:1:2 in $100 \mathrm{~mL}$ of $2 \mathrm{M}$ hydrochloric acid was heated in an oil bath at $120{ }^{\circ} \mathrm{C}$ for $6 \mathrm{~h}$ with occasional shaking. The resinous product so obtained was repeatedly washed with cold distilled water dried in air and powdered with the help of agate mortar and pestle. The powder was washed many times with hot water to remove unreacted monomers. The air dried powder was extracted with diethyl ether and then petroleum ether was used to remove salicylic acid-diaminonaphthalene and other possible copolymers, which might be present along with SDNF terpolymer. It was further purified by dissolving in $8 \%$ sodium hydroxide solution, filtered and reprecipited by gradual drop wise addition of $1: 1(\mathrm{v} / \mathrm{v})$ hydrochloric acid with constant and rapid stirring to avoid lump formation. The SDNF terpolymer resin so obtained was filtered washed several times with hot water dried and purity checked with thin layer chromatography (TLC) technique $e^{12,13}$.

It is then characterized by elemental analysis, infrared (IR) spectroscopy, nuclear magnetic resonance (NMR) spectroscopy and UV-Visible spectral studies. The newly synthesized terpolymer resin is soluble in dimethylformamide (DMF), dimethylsulfoxide (DMSO), aqueous $\mathrm{NaOH}$. However, it is insoluble in acids and common organic solvents. The number average molecular weight of the resin was determined by non-aqueous conductometric titration. The structure of polymer is given below (Scheme 1).
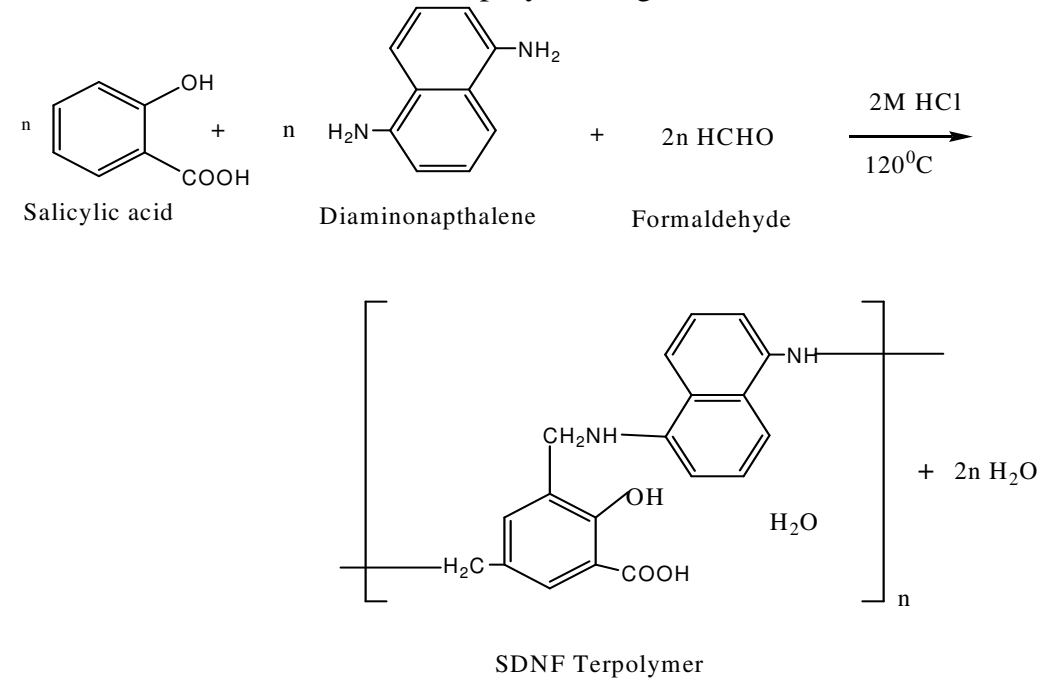

\section{Thermogravimetry}

Scheme 1.

Thermal analysis method is associated with a change in weight with respect to temperature. Heating is performed under strictly controlled conditions and can reveal changes in structure and other important properties of the material being studied. In non-isothermal or dynamic TGA the sample is subjected to conditions increase in temperature at linear rate ${ }^{14-15}$. 
Thermogravimetric analyses (TGA) of terpolymer sample have been carried out by using Perkins Elmer TGS-ll thermal analyzer at heating rate of $10{ }^{\circ} \mathrm{C}$ per minute and in air atmosphere up to $800{ }^{\circ} \mathrm{C}$. The thermograms were recorded at Sophisticated Instrumentation Centre for Applied Research and Testing (SICART), Vallabh Vidyanagar, Gujrat.

The Freeman - Carroll and Sharp- Wentworth methods have been employed for the calculation of kinetic parameters of the newly synthesized terpolymer resin with help of dynamic TG curve ${ }^{11-13}$. The advantage of Freeman and Carroll method that in one single stage by keeping heating rate constant both the order of reaction and energy of activation can calculated in a single experiment. The following expression is used to evaluate various kinetic parameters:

$$
\frac{\Delta \log \left(\frac{d w}{d t}\right)}{\Delta \log W_{r}}=-\left[\frac{E}{2.303 R}\right] \frac{\Delta(1 / T)}{\Delta \log W_{r}}+n
$$

Hence, a plot of $\frac{\Delta \log \left(\frac{d w}{d t}\right)}{\Delta \log W_{r}}$ vs. $\frac{\Delta(1 / T)}{\Delta \log W_{r}}$ should give a straight line with an intercept on $y$-axis equal to the value of $\mathrm{n}$ (the order of reaction) and the slope $\mathrm{m}=\mathrm{E} / 2.303 \mathrm{R}$. Where, $\mathrm{dw} / \mathrm{dt}$ is the rate of change of weight with time and in expression $\mathrm{Wr}=\mathrm{Wc}-\mathrm{W}, \mathrm{Wc}$ is the weight loss at the completion of the reaction, $w$ is the total weight loss up to the time $t$ and $\mathrm{T}$ is the temperature in $\mathrm{k}$.

The following expression is used to evaluate Ea with Sharp- Wentworth method:

$$
\log \frac{(d c / d T)}{(1-c)}=\log (A / \beta)-\left[\frac{E_{a}}{2.303 R}\right] \cdot \frac{1}{T}
$$

Where, $\mathrm{dc} / \mathrm{dt}$ is the rate of change of mass with time $\mathrm{t}, \mathrm{T}$ is the temperature and $\beta=\Delta \mathrm{T} / \mathrm{dt}$.

\section{Results and Discussion}

The Thermal degradation curve for SDNF resin is shown in Figure 1 exhibits four-stage decomposition and ranges are given in Table 1. The first stage decomposition which was slow and ranged from $40-160{ }^{\circ} \mathrm{C}$ corresponding to loss $7.3 \%$ which may have been due to entrapped $\mathrm{H}_{2} \mathrm{O}$ molecule. The second stage decomposition represents degradation of side chain attached to aromatic nucleus and - $\mathrm{COOH}$ group. The observed mass loss is $47.5 \%$ against calculated $46.3 \%$. The third stage decomposition at $250-370{ }^{\circ} \mathrm{C}$ is due to the loss of phenolic -OH group observed $62.4 \%$ and calculated $63.5 \%$. The fourth state decomposition corresponds to total decomposition of terpolymer resin. The Half Decomposition temperature for SDNF terpolymer resin is found to be $282^{\circ} \mathrm{C}$.

Table 1. Thermoanalytical data and decomposition temperature of SDNF terpolymer resin.

\begin{tabular}{cccccc}
\hline Terpolymer & $\begin{array}{c}\text { Temperature } \\
\text { Range }{ }^{\circ} \mathrm{C}\end{array}$ & $\begin{array}{c}\text { Stage of } \\
\text { Decomposition }\end{array}$ & Species Degraded & \multicolumn{2}{c}{$\%$ Weight loss } \\
\cline { 5 - 6 } SDNF & $40-80$ & First & $\begin{array}{c}\text { Loss of entrapped } \\
-\mathrm{H}_{2} \text { O molecule }\end{array}$ & 5.0 & 5.3 \\
& $80-210$ & Second & $\begin{array}{c}\text { Loss of side chain } \\
\text { attached to } \\
\text { aromatic nucleus. } \\
\text { Loss of -COOH } \\
\text { group. }\end{array}$ & 49.3 & 48.5 \\
\hline Chemplete & 60.0 & 61.8 \\
\hline
\end{tabular}




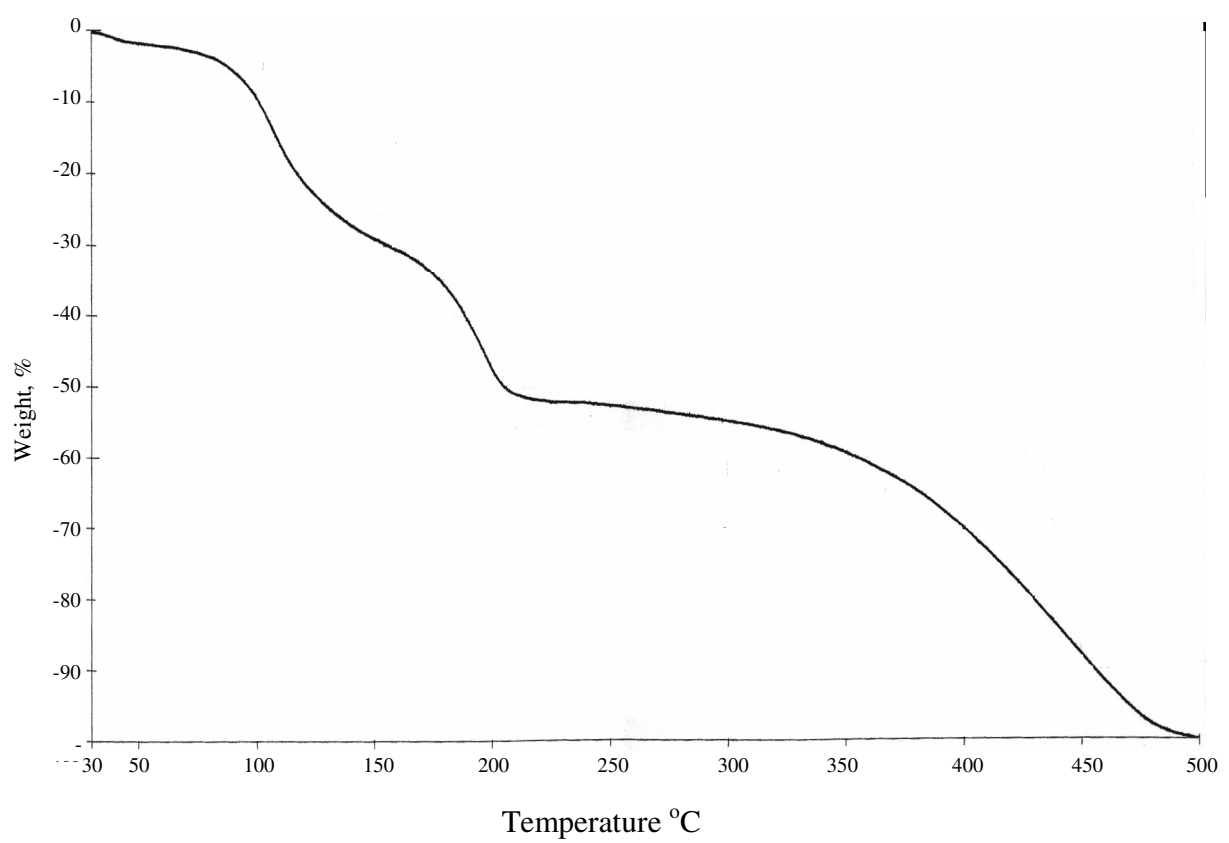

\section{Conclusion}

Figure 1. Thermogram of SDNF terpolymer resin.

Thermogram of SDNF terpolymer resin shows activation energy calculated by the Freeman - Carroll and Sharp- Wentworth methods are in good agreement with each other. Thermodynamic parameters have been calculated on the basis of thermal activation energy and values are given in Table 2. Due to abnormally low value of frequency factor $[Z]$ it may be classified as a slow reaction and no other obvious reason can be given. The value of entropy $[\Delta \mathrm{S}]$ indicates that the activated polymer has more ordered structure than the reactants and the reaction are slower than normal. This is further supported by low $\mathrm{Z}$ values ${ }^{13-15}$.t is very difficult to draw any unique conclusion from the magnitude of thermal activation energy [Ea] as decomposition mechanism is expected to be complicated. Positive values of activation energy under present investigation correspond to the energy of activation due oxidation -reduction process of terpolymer in the higher temperature range ${ }^{13-16}$.

Table 2. Result of thermogravimetric analysis of SDNF terpolymer resin.

\begin{tabular}{|c|c|c|c|c|c|c|c|c|c|}
\hline \multirow[b]{2}{*}{ 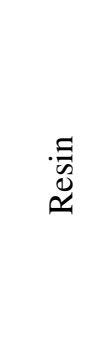 } & \multirow[b]{2}{*}{ 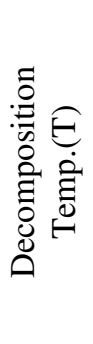 } & \multirow[b]{2}{*}{ 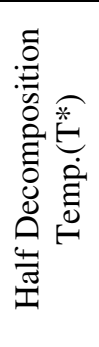 } & \multicolumn{2}{|c|}{$\begin{array}{c}\text { Activation } \\
\text { Energy } \mathrm{J} / \mathrm{mole}\end{array}$} & \multicolumn{5}{|c|}{ Kinetic parameters by FC } \\
\hline & & & 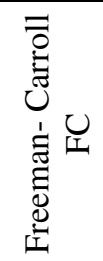 & 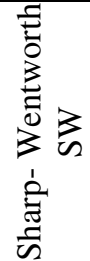 & 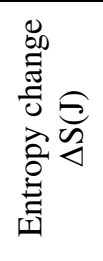 & 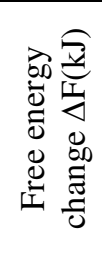 & 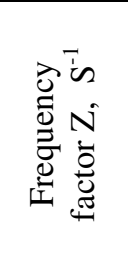 & 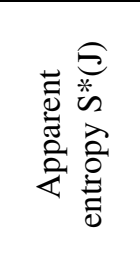 & $=$ \\
\hline SDNF & 178 & 205 & 16.40 & 15.1 & 6.63 & 15.04 & 152.854 & -24.6566 & 1.08 \\
\hline
\end{tabular}


Fairly straight-line plots are obtained using the two methods. However, using the Freeman- Carroll method some abnormal points were ignored to get a clear picture about most of the points. Similarly, in the Sharp- Wentworth method, some points at the beginning or the end did not fall on straight line. This is expected, since, the decomposition of terpolymer is not obeying first order kinetics perfectly. These observations are in harmony with the findings of Jacobs and Tompkin and other earlier workers ${ }^{17}$

\section{Aknowledgement}

The authors are thankful to the Head of the Department of Chemistry, Rashtrasant Tukadoji Maharaj Nagpur University, Nagpur for providing necessary laboratory facilities.

\section{References}

1. Jadhao M, Paliwal L J and Bhave N S, XIV National Symposium \& Workshop Vadodara, India Thermans 2004, 2004, 250-253

2. Das A P, Lenka S and Nayak P L, J Appl Polym Sci., 1985, 30, 4619.

3. Samal R K and Senapati B K, J Appl Polym Sci., 1996, 62, 655.

4. DeGeiso R.C, Donaruma L G and Tomic E A, Anal Chem., 1962, 34, 845.

5. Michael P E, Lingala P S, Juneja H D and Paliwal L J, J Appl Polym Sci., 2004, 92, 2278.

6. Gurnule W B, Juneja H D and Paliwal L J, Asian J Chem., 1999, 11(3), 767.

7. Lingala P S, Juneja H D and Paliwal L J, Proc. of the $12^{\text {th }}$ National Symposium on Thermal Analysis, Thermans 2000, Gorkhpur, 2000, pp 245-247.

8. Manvalan R and Patel M M, J Mackromol Chem., 1983, A-20(9), 907-925.

9. Freeman E.S and Carroll B, J Phys Chem., 1958, 62, 394.

10. Freeman E S and Anderson D A, J Polym Sci., 1961, 54, 253.

11. Sharp J B and Wentworth S A, Anal Chem., 1969, 41, 2060.

12. Masram D T, Kariya K P and Bhave, N S, E-Polymer, 2007, 075,.

13. Jadhao M, Paliwal L J and Bhave N S, J Appl Polym Sci., 2005, 96, 1605.

14. Coats A W and Redfen J P, Nature, 1964, 201, 68.

15. Ozawa T, J Thermal Analysis, 1985, 7, 601.

16. Pal T K and Kharat R B, Indian J Chem., 1989, 28-A, 55-58.

17. Jacobs P W M, F C Tompkins "Chemistry of Solids State", E.G Garner Publication, London, 1955,188. 


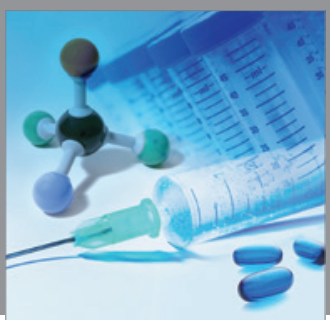

International Journal of

Medicinal Chemistry

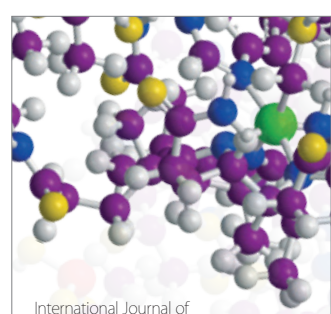

Carbohydrate Chemistry

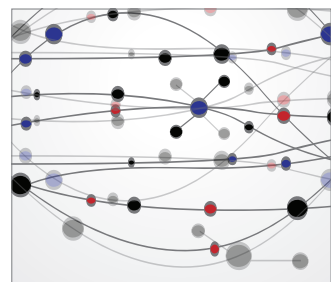

The Scientific World Journal
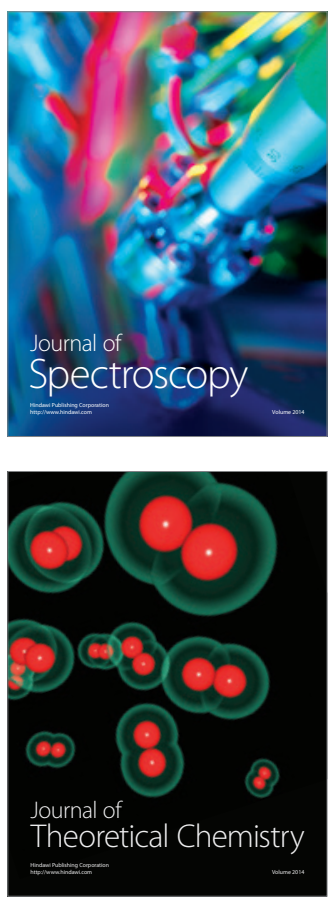
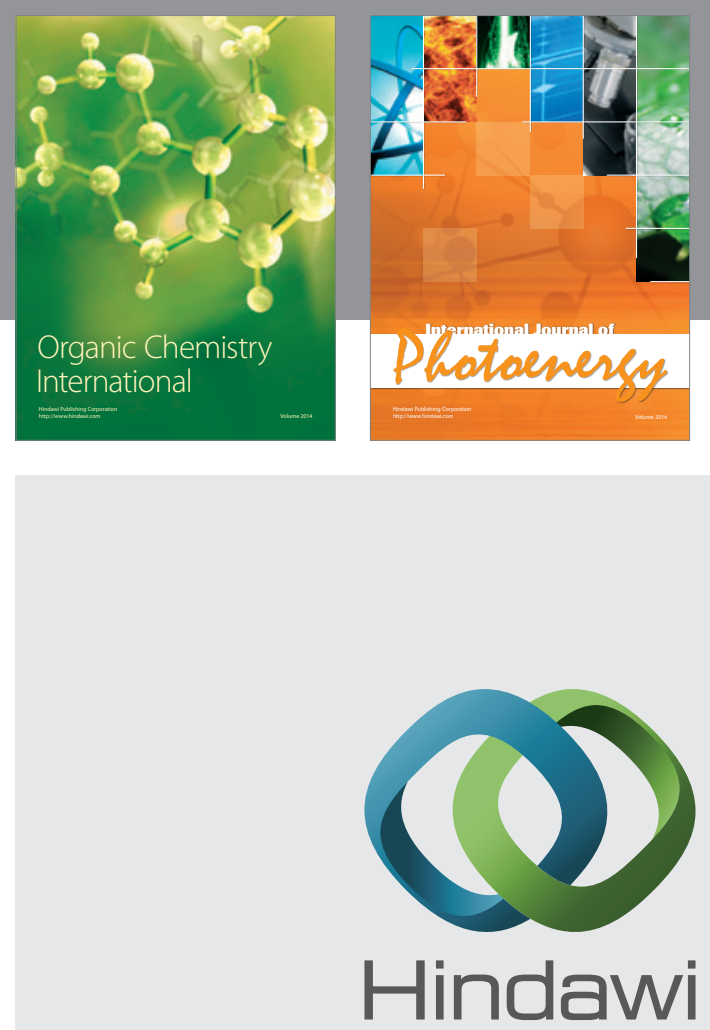

Submit your manuscripts at

http://www.hindawi.com
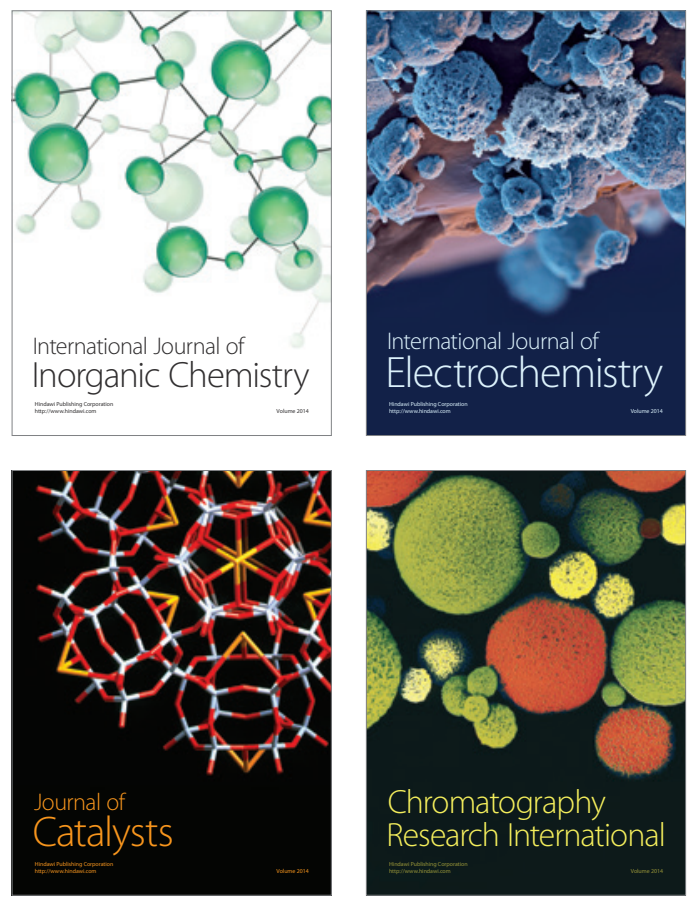
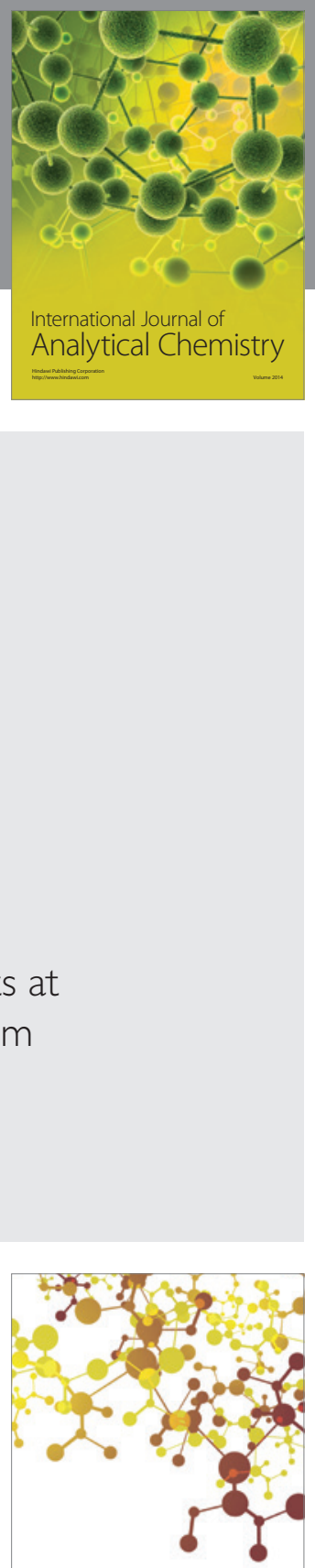

Journal of

Applied Chemistry
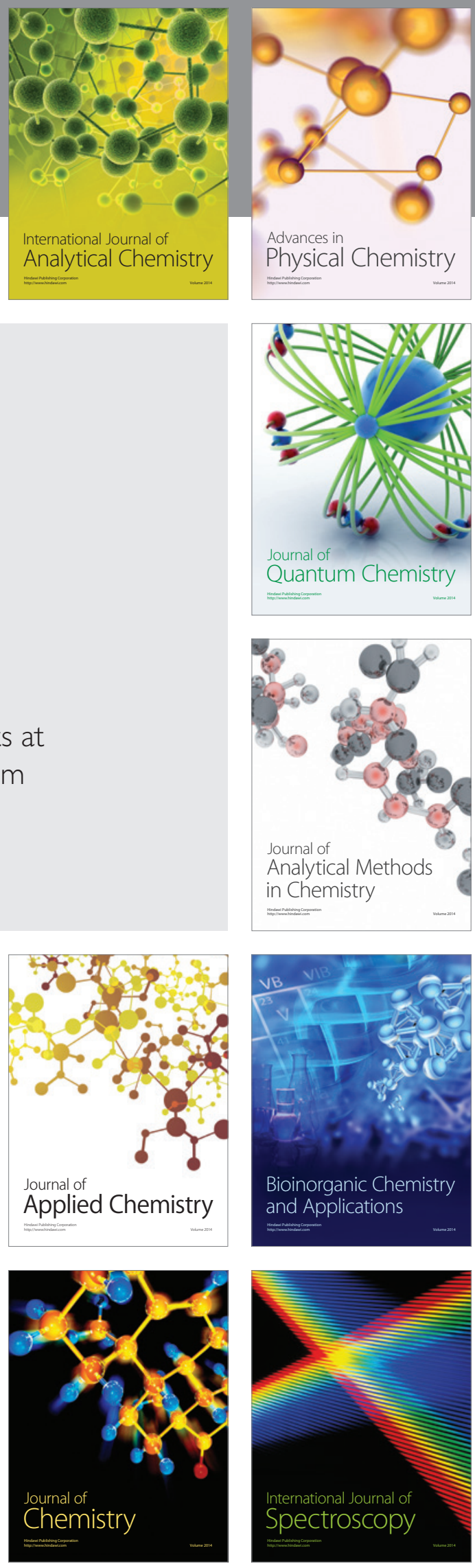